ESAIM: COCV 27 (2021) 59

https://doi.org/10.1051/cocv/2021047
ESAIM: Control, Optimisation and Calculus of Variations

www.esaim-cocv.org

\title{
OPTIMAL ENERGY DECAY RATES FOR ABSTRACT SECOND ORDER EVOLUTION EQUATIONS WITH NON-AUTONOMOUS DAMPING*
}

\author{
Jun-Ren LuO ${ }^{1,2}$ AND Ti-Jun XIAO ${ }^{2, * *}$
}

\begin{abstract}
We consider an abstract second order non-autonomous evolution equation in a Hilbert space $H: u^{\prime \prime}+A u+\gamma(t) u^{\prime}+f(u)=0$, where $A$ is a self-adjoint and nonnegative operator on $H, f$ is a conservative $H$-valued function with polynomial growth (not necessarily to be monotone), and $\gamma(t) u^{\prime}$ is a time-dependent damping term. How exactly the decay of the energy is affected by the damping coefficient $\gamma(t)$ and the exponent associated with the nonlinear term $f$ ? There seems to be little development on the study of such problems, with regard to non-autonomous equations, even for strongly positive operator $A$. By an idea of asymptotic rate-sharpening (among others), we obtain the optimal decay rate of the energy of the non-autonomous evolution equation in terms of $\gamma(t)$ and $f$. As a byproduct, we show the optimality of the energy decay rates obtained previously in the literature when $f$ is a monotone operator.
\end{abstract}

Mathematics Subject Classification. 35B35, 93D20, 34G20, 35L70, 35L90.

Received July 23, 2020. Accepted April 22, 2021.

\section{INTRODUCTION}

In view of the importance in mathematical theory and applications in physics, engineering, mechanics, biology and others, various nonautonomous equations including the non-autonomous evolution equations in abstract (infinite-dimensional) spaces, have been studied by many researchers and a lot of good results on this issue have been established (cf., e.g. [1, 3, 8-17, 24-26, 28, 30, 32, 34, 35] and references therein).

In this paper, we are concerned with an abstract second order non-autonomous evolution equation in a real Hilbert space $H$, with time dependent damping, as follows

$$
\left\{\begin{array}{l}
u^{\prime \prime}(t)+A u(t)+\gamma(t) u^{\prime}(t)+f(u(t))=0, \quad \forall t \geq 0, \\
u(0)=u_{0}, u^{\prime}(0)=u_{1},
\end{array}\right.
$$

* The work was supported partly by the NSF of China $(11771091,11831011)$, and the Shanghai Key Laboratory for Contemporary Applied Mathematics (08DZ2271900).

Keywords and phrases: Non-autonomous, abstract second order evolution equation, time dependent damping, energy estimates, slow solutions, nonlinear source, Hilbert space.

${ }^{1}$ College of Science, University of Shanghai for Science and Technology, Shanghai 200093, PR China.

2 Shanghai Key Laboratory for Contemporary Applied Mathematics, School of Mathematical Sciences, Fudan University, Shanghai 200433, PR China.

** Corresponding author: tjxiao@fudan.edu.cn 
where $A$ is a nonnegative self-adjoint linear operator on $H$, the nonlinear term $f: D\left(A^{1 / 2}\right) \rightarrow H$ is assumed to be conservative with polynomial growth, and $\gamma u^{\prime}$ is the time dependent damping with

$$
R_{1}(1+t)^{-\alpha_{1}} \leq \gamma(t) \leq R_{2}(1+t)^{-\alpha_{2}}, \quad \forall t \geq 0,
$$

where $R_{1}$ and $R_{2}$ are two positive constants, and $0 \leq \alpha_{2} \leq \alpha_{1}<1$.

For the study of the asymptotic behaviors and decay rates for the damped autonomous evolution equations, there have been many developments. We refer the reader to, e.g., $[2,4,6,12,13,15,17-21,27,31,35]$ and references therein. Especially, when $A$ is strongly positive, the asymptotic behaviors for the autonomous case of (1.1) have been extensively studied too, $c f$. [10, 22, 23, 29, 30, 32, 33]. In this paper, we assume $A$ to just have a nontrivial kernel; for the case of $\gamma(t)$ being constant, optimal decay rates have been obtained, and fast and slow solutions are classified subtly (see [18-20]).

It was shown in [7] that any bounded solution of (1.1) converges weakly in $H_{1}$ to a stationary point of the potential energy, when $f$ is a general monotone operator. Following the work, it was proved in [31] that any solution energy $E(t)$ satisfies that for every $\bar{\alpha}<\alpha_{1}$,

$$
E(t)=\mathrm{o}\left(\frac{1}{t^{1+\bar{\alpha}}}\right) \text { as } t \rightarrow+\infty
$$

under the condition that $\gamma(t)$ is decreasing,

$$
\gamma(t) \geq c(1+t)^{-\alpha_{1}}, \quad t \geq 0
$$

(with a constant $c>0$ ), and

$$
\gamma^{\prime}(t) \leq-\alpha_{1} \frac{\gamma(t)}{1+t}, \quad t \geq t_{0} \geq 0 \text { a.e. }
$$

We note that the two inequalities (1.4) and (1.5) together imply that

$$
\gamma(t) \leq C(1+t)^{-\alpha_{1}}, \quad t \geq 0
$$

(with a constant $C>0$ ). For

$$
\gamma(t)=(1+t)^{-\alpha} \quad(\alpha \in[0,1)),
$$

the estimate (1.3) was later improved in [4] as

$$
E(t)=\mathrm{o}\left(\frac{1}{t^{1+\alpha}}\right) \text { as } t \rightarrow+\infty .
$$

However, what is the optimal decay rate of the energy of the non-autonomous equation (1.1), i.e., how exactly the decay of the energy of (1.1) is affected by the damping coefficient $\gamma(t)$ and the exponent associated with the nonlinear term $f$ ? This problem is still unsolved. To the best of our knowledge, there has been little development so far on the study of such problems, with regard to non-autonomous equations, even for strongly positive operator $A$; see Section 1.3 of [30] about an optimality result for one dimensional wave equation damped by a time-dependent boundary feedback, whose proof relies on d'Alembert's formula.

In this paper, we devote ourselves to studying the problem. 
Let $\gamma(t)$ be as in (1.6), and $f(u)=|u|^{p} u(p>0)$. By Theorem 2.4 of [28], the energy has the upper estimate

$$
E(t) \leq C(1+t)^{-(1-\alpha)(p+2) / p}
$$

On the other hand, to get a lower bound we may exploit the hyperbolic version of the Dirichlet quotient

$$
G(t):=\frac{\left\|u^{\prime}(t)\right\|^{2}+\left\|A^{1 / 2} u(t)\right\|^{2}}{\|u(t)\|^{2 p+2}}
$$

introduced in [18]. Estimating the time-derivative of $\hat{G}(t)$, a small perturbation of $G$ with $\gamma$ as a product factor (see (4.9), Sect. 4), with the aid of either (1.7) or (1.8) we might probably obtain a lower estimate

$$
E(t) \geq c(1+t)^{-(1+\alpha)(p+2) / p}
$$

for a nonempty open set of initial data, but under the restriction

$$
\alpha<p /(p+4) \text { or } \quad \alpha<1 / 3 \text {. }
$$

Obviously, there is a gap between the exponents $-(1-\alpha)(p+2) / p$ and $-(1+\alpha)(p+2) / p$, when $\alpha>0$. Actually, it is still unknown what is the best possible upper estimate.

By an idea of asymptotic rate-sharpening, we obtain the optimal decay rate of the energy of this abstract second order non-autonomous evolution equation in terms of $\gamma(t)$ and $f$. Moreover, as a byproduct, we show that, when $\mathrm{f}$ is a monotone operator, some energy decay rates given previously in the literature are optimal.

More explicitly, we obtain upper estimates for the solutions and their energies of (1.1) in a general setting, and single out slow solutions (decaying exactly at the rates corresponding to the upper estimates) with a nonempty open set of initial data. When $\gamma(t)$ is a constant, the estimates recover those given in Theorems 2.2 and 2.3 of [18]. Moreover, specialized to the case of (1.6), the exponents of the upper and lower bounds coincide and read $-(1+\alpha)(p+2) / p$ for all $\alpha \in[0,1)$. In addition, since

$$
-(1+\alpha)(p+2) / p \rightarrow-(1+\alpha) \text { as } p \rightarrow+\infty,
$$

we see that the decay rate in (1.7) is the best possible for a general monotone $f$ (as considered in $[4,7,31]$ ).

The outline of this paper is the following. In Section 2 we state assumptions and present our main theorems. Section 3 is devoted to formulating several preliminary results. We employ these results in Section 4 to prove the main theorems. Finally, in Section 5 we give two examples of our theorems being applied to Neumann or Dirichlet problems for nonautonomous, semilinear wave equations.

\section{MAIN THEOREMS}

We denote by $\langle v, w\rangle$ the inner product of two vectors $v, w$ in $H$, and by $\|v\|$ the $H$-norm of $v$, and we define

$$
H_{1}=D\left(A^{1 / 2}\right) \quad \text { with norm }\|v\|_{H_{1}}:=\left(\|v\|^{2}+\left\|A^{1 / 2} v\right\|^{2}\right)^{1 / 2} .
$$

The following is the basic assumptions on $\gamma$ and $f$.

\section{Assumption (H1)}

(i) $\gamma \in W_{\text {loc }}^{1, \infty}\left(R^{+}\right)$satisfies $(1.2)$;

(ii) $f=\nabla F: H_{1} \rightarrow H$ is a locally Lipschitz continuous gradient operator of some nonnegative functional $F$ on $H_{1}$, with $F(0)=0$. 
One knows

$$
F(v)=\int_{0}^{1}\langle f(t v), v\rangle \mathrm{d} t, \quad \forall v \in H_{1}
$$

A function $u \in C\left([0,+\infty) ; H_{1}\right) \cap C^{1}([0,+\infty) ; H)$ is called a mild solution of problem (1.1), if it satisfies the integral equation

$$
u(t)=S^{\prime}(t) u_{0}+S(t) u_{1}-\int_{0}^{t} S(t-\tau)\left[\gamma(\tau) u^{\prime}(\tau)+f(u)\right] \mathrm{d} \tau, \quad t \geq 0 .
$$

Here $S(\cdot):[0,+\infty) \rightarrow \mathcal{L}(H)$ (the space of bounded linear operators on $H$ ) is a solution operator for the linear equation

$$
u^{\prime \prime}(t)+A u(t)=0, \quad t \geq 0,
$$

with $S(0)=0$ and $S^{\prime}(0)=I$ (the identity; the derivative being in the sense of strong topology).

In particular, $u$ is called a strong solution if $u \in C^{1}\left([0,+\infty) ; H_{1}\right) \cap C^{2}([0,+\infty) ; H)$ and $(1.1)$ holds.

The following result concerning the wellposedness of (1.1) is from Proposition 2.3 of [28].

Proposition 2.1. Assume (H1). Then for every $\left(u_{0}, u_{1}\right) \in H_{1} \times H$, problem (1.1) admits a unique mild solution $u$, which depends continuously on the initial data. In particular, $u$ is a strong solution if $\left(u_{0}, u_{1}\right) \in D(A) \times H_{1}$. Moreover, defining the energy

$$
E(t):=\frac{1}{2}\left\|u^{\prime}(t)\right\|^{2}+\frac{1}{2}\left\|A^{1 / 2} u(t)\right\|^{2}+F(u(t)), \quad t \geq 0,
$$

one has

$$
\frac{\mathrm{d} E(t)}{\mathrm{d} t}=-\gamma(t)\left\|u^{\prime}(t)\right\|^{2}, \quad t \geq 0
$$

(for strong solutions).

For energy decay, more conditions are required.

\section{Assumption (H2)}

(1) For $v \in H_{1}$,

$$
\langle f(v), v\rangle \geq c F(v) \geq 0,
$$

with some constant $c>0$;

(2) for $v \in H_{1}$,

$$
\|v\|^{p+2} \leq M_{0}\left(\left\|A^{1 / 2} v\right\|\right)\left(\left\|A^{1 / 2} v\right\|^{2}+F(v)\right)
$$

with constant $p>0$ and some positive function $M_{0}$ on $R_{+}$that is bounded on bounded sets;

(3) for $t \geq 0$,

$\left|\gamma^{\prime}(t)\right| \leq C \gamma^{2}(t)$ with some constant $C>0$, or $\alpha_{1}=\alpha_{2} ;$

(4) for $t \geq 0$,

$\left|\gamma^{\prime}(t)\right| \leq C \gamma(t)(1+t)^{-1}$ with some constant $C>0$ or $\gamma^{\prime}(t) \leq 0$, in the case $\alpha_{1}>1 / 2$; 
(5) $2 \alpha_{1}<1+\alpha_{2}$, in the case $\alpha_{1}>1 / 2$.

Now, we present our first main result.

Theorem 2.2. Let Assumptions (H1) and (H2) hold. Let $\left(u_{0}, u_{1}\right) \in H_{1} \times H$, and let $u(t)$ be the unique global mild solution of problem (1.1). Then

$$
\begin{aligned}
& E(t) \leq M_{1}(E(0))\left(1+\int_{0}^{t} \gamma(\tau)^{-1} \mathrm{~d} \tau\right)^{-\frac{p+2}{p}}, \quad \forall t \geq 0, \\
& \|u(t)\| \leq M_{2}(E(0))\left(1+\int_{0}^{t} \gamma(\tau)^{-1} \mathrm{~d} \tau\right)^{-\frac{1}{p}}, \quad \forall t \geq 0,
\end{aligned}
$$

for some positive functions $M_{1}$ and $M_{2}$ on $R_{+}$that are bounded on bounded sets.

For the existence of slow solutions, we need additional conditions.

\section{Assumption (H3)}

(1) $\operatorname{ker} A \neq\{0\}$, and there exists $\zeta>0$ such that

$$
\left\|A^{1 / 2} u\right\|^{2} \geq \zeta\|u\|^{2}, \quad \forall u \in H_{1} \cap(\operatorname{ker} A)^{\perp} ;
$$

(2) there exist positive number $\xi$ and $M$ such that

$$
\|f(u)\| \leq M\left(\|u\|^{1+p}+\left\|A^{1 / 2} u\right\|^{1+p}\right), \quad \forall u \in H_{1} \text { with }\|u\|_{H_{1}} \leq \xi
$$

(3) $\gamma(t) \gamma(s)^{-1} \leq C_{0}, \quad \forall t \geq s \geq 0$, with some constant $C_{0}>0$.

The following is our second main result.

Theorem 2.3. Let Assumptions (H1)-(H3) hold. Then there exists a nonempty open set $S \subset H_{1} \times H$ such that for every $\left(u_{0}, u_{1}\right) \in S$, the unique global solution of problem (1.1) satisfies

$$
\begin{aligned}
& \|u(t)\| \geq c_{0}\left(1+\int_{0}^{t} \gamma(\tau)^{-1} \mathrm{~d} \tau\right)^{-\frac{1}{p}}, \quad \forall t \geq 0, \\
& E(t) \geq c_{0}\left(1+\int_{0}^{t} \gamma(\tau)^{-1} \mathrm{~d} \tau\right)^{-\frac{p+2}{p}}, \quad \forall t \geq 0,
\end{aligned}
$$

for some positive function $c_{0}$ depending on $\left\|u_{0}\right\|,\left\|A^{1 / 2} u_{0}\right\|$ and $\left\|u_{1}\right\|$.

Remark 2.4. (1) When $\gamma$ is decreasing and $\alpha_{1}=\alpha_{2}=\alpha$, we know that (H2)(3)-(5) and (H3)(3) are satisfied, and the upper estimate of the energy in (2.3) reads

$$
E(t) \leq M_{1}(E(0))(1+t)^{-(1+\alpha)(p+2) / p}, \quad \forall t \geq 0,
$$


and the lower estimate in (2.4) reads

$$
E(t) \geq c_{0}(1+t)^{-(1+\alpha)(p+2) / p}, \quad \forall t \geq 0
$$

(2) The inequality $\left|\gamma^{\prime}(t)\right| \leq C \gamma^{2}(t)$ in (H2)(3) is weaker than $\left|\gamma^{\prime}(t)\right| \leq C \gamma(t)(1+t)^{-1}$ in (H2)(4).

(3) The inequality $2 \alpha_{1}<1+\alpha_{2}$ (in (H2)(5)) holds automatically, when $\alpha_{1} \in[0,1 / 2)$.

(4) Condition $(\mathrm{H} 3)(2)$ is stronger than the corresponding one in $[18,(2.14)]$ :

$$
\|f(u)\| \leq M\left(\|u\|^{1+p}+\left\|A^{1 / 2} u\right\|^{1+\beta}\right), \quad \forall u \in H_{1} \text { with }\|u\|_{H_{1}} \leq \xi,
$$

where $M, \xi, \beta$ are positive constants. It remains open whether condition (H3)(2) in Theorem 2.3 can be improved as (2.5).

\section{Preliminary Results AND Proofs}

Throughout this section, $\bar{C}, \tilde{C}, C_{1}, C_{2}, \ldots, C_{17}$ denote positive constants depending on the values of $E(0)$ and being bounded on bounded sets of the values, and they may be different at different positions.

From (2.2),

$$
\frac{1}{2}\left\|u^{\prime}(t)\right\|^{2}+\frac{1}{2}\left\|A^{1 / 2} u(t)\right\|^{2} \leq E(t) \leq E(0),
$$

for strong solutions (and so for mild solution as well, by a density argument). Thus, from (H2)(2) we have

$$
\|u\|^{p+2} \leq \bar{C}(E(0)) E(t), \quad \forall u \in H_{1}, t \geq 0,
$$

for some positive function $\bar{C}$ on $R_{+}$that is bounded on bounded sets.

Now, we establish an important lemma with respect to a perturbed energy functional $E_{\varepsilon, \eta}(t)$ (see below), which is useful in the proof of Theorem 2.2. The functional with $\eta(t)=1$ was employed in [18] to establish the upper estimate of the energy when $\gamma(t)=1$.

Lemma 3.1. Assume (H1) and (H2)(1). Let $u(t)$ be a strong solution of (1.1), and let $\eta \in W_{\mathrm{loc}}^{1, \infty}\left(R^{+}\right)$be a positive function, $\varepsilon \in(0,1)$, and

$$
r:=\frac{p}{p+2}
$$

Define

$$
E_{\varepsilon, \eta}(t):=E(t)+\varepsilon \eta(t)[E(t)]^{r}\left\langle u^{\prime}(t), u(t)\right\rangle .
$$

Then

$$
E_{\varepsilon, \eta}^{\prime}(t) \leq-\frac{1}{2} \gamma(t)\left\|u^{\prime}(t)\right\|^{2}+\varepsilon \tilde{C}(E(0)) V_{1}(t) \eta(t)\left\|u^{\prime}(t)\right\|^{2}+\varepsilon\left[\varepsilon \tilde{C}(E(0)) V_{2}(t)-\tilde{c}\right] \eta(t)[E(t)]^{r+1}, \quad t \geq 0,
$$

with some $\tilde{c} \in(0,1)$ and some positive function $\tilde{C}$ that are bounded on bounded sets. Here,

$$
V_{1}(t):=[E(t)]^{\frac{p}{2 p+4}} \gamma(t)+[E(t)]^{r},
$$




$$
V_{2}(t):=\gamma(t) \eta(t)\left[1+\left(\frac{\eta^{\prime}(t)}{\eta(t) \gamma(t)}\right)^{2}\right]
$$

Proof. The time-derivative of $E_{\varepsilon, \eta}(t)$ is

$$
\begin{aligned}
E_{\varepsilon, \eta}^{\prime}(t)= & -\gamma(t)\left\|u^{\prime}\right\|^{2}-\varepsilon r \eta(t)[E(t)]^{r-1} \gamma(t)\left\langle u^{\prime}, u\right\rangle\left\|u^{\prime}\right\|^{2}+\varepsilon \eta(t)[E(t)]^{r}\left\|u^{\prime}\right\|^{2} \\
& -\varepsilon \eta(t)[E(t)]^{r} \gamma(t)\left\langle u^{\prime}, u\right\rangle-\varepsilon \eta(t)[E(t)]^{r}\left(\left\|A^{1 / 2} u(t)\right\|^{2}+\langle f(u), u\rangle\right) \\
& +\varepsilon \eta^{\prime}(t)[E(t)]^{r}\left\langle u^{\prime}, u\right\rangle \\
= & : I_{1}+I_{2}+I_{3}+I_{4}+I_{5}+I_{6} .
\end{aligned}
$$

We first estimate $I_{2}$. By (3.1) and the Cauchy-Schwarz inequality we obtain

$$
r[E(t)]^{r-1}\left\langle u^{\prime}, u\right\rangle \leq r[E(t)]^{r-1}\left\|u^{\prime}\right\|\|u\| \leq C_{1}[E(t)]^{r-1+\frac{1}{2}+\frac{1}{p+2}}=C_{1}[E(t)]^{\frac{p}{2 p+4}},
$$

with a constant $C_{1}>0$. Hence

$$
I_{2} \leq C_{1} \varepsilon \eta(t)[E(t)]^{\frac{p}{2 p+4}} \gamma(t)\left\|u^{\prime}\right\|^{2}
$$

For $I_{4}$, we use Young's inequality, as well as the Cauchy-Schwarz inequality and (3.1) to infer that

$$
\begin{aligned}
\varepsilon \eta(t)[E(t)]^{r} \gamma(t)\left\langle u^{\prime}, u\right\rangle & \leq \frac{1}{4} \gamma(t)\left\|u^{\prime}\right\|^{2}+\gamma(t) \varepsilon^{2} \eta^{2}(t)[E(t)]^{2 r}\|u\|^{2} \\
& \leq \frac{1}{4} \gamma(t)\left\|u^{\prime}\right\|^{2}+C_{2} \varepsilon^{2} \gamma(t) \eta^{2}(t)[E(t)]^{2 r+\frac{2}{p+2}}
\end{aligned}
$$

Since $2 r+\frac{2}{p+2}=r+1$, this means that

$$
I_{4} \leq \frac{1}{4} \gamma(t)\left\|u^{\prime}\right\|^{2}+C_{2} \varepsilon^{2} \gamma(t) \eta^{2}(t)[E(t)]^{r+1}
$$

Similarly, we have

$$
I_{6} \leq \frac{1}{4} \gamma(t)\left\|u^{\prime}\right\|^{2}+C_{3} \varepsilon^{2} \gamma(t)^{-1}\left[\eta^{\prime}(t)\right]^{2}[E(t)]^{r+1}
$$

Moreover, in view of $(\mathrm{H} 2)(1)$ and (2.1) we deduce that

$$
\begin{aligned}
{[E(t)]^{r}\left(\left\|A^{1 / 2} u(t)\right\|^{2}+\langle f(u), u\rangle\right) } & \geq c_{1}[E(t)]^{r}\left(\frac{1}{2}\left\|A^{1 / 2} u(t)\right\|^{2}+F(u)\right) \\
& =c_{1}[E(t)]^{r}\left(E(t)-\frac{1}{2}\left\|u^{\prime}\right\|^{2}\right) \\
& =c_{1}[E(t)]^{r+1}-\frac{c_{1}}{2}[E(t)]^{r}\left\|u^{\prime}\right\|^{2},
\end{aligned}
$$

with some constant $c_{1}>0$, so that

$$
I_{5} \leq-c_{1} \varepsilon \eta(t)[E(t)]^{r+1}+\frac{c_{1}}{2} \varepsilon[E(t)]^{r} \eta(t)\left\|u^{\prime}\right\|^{2}
$$


Finally, combining (3.3)-(3.6) together we have

$$
\begin{aligned}
E_{\varepsilon, \eta}^{\prime}(t) \leq & -\frac{1}{2} \gamma(t)\left\|u^{\prime}\right\|^{2}+C_{1} \varepsilon \eta(t)[E(t)]^{\frac{p}{2 p+4}} \gamma(t)\left\|u^{\prime}\right\|^{2}+\frac{c_{1}}{2} \varepsilon \eta(t)[E(t)]^{r}\left\|u^{\prime}\right\|^{2} \\
& +C_{2} \varepsilon^{2} \gamma(t) \eta^{2}(t)[E(t)]^{r+1}-c_{1} \varepsilon \eta(t)[E(t)]^{r+1}+C_{3} \varepsilon^{2} \gamma(t)^{-1}\left[\eta^{\prime}(t)\right]^{2}[E(t)]^{r+1} .
\end{aligned}
$$

This gives the estimate of $E_{\varepsilon, \eta}^{\prime}(t)$ as required.

Next, we derive a preliminary upper estimate of energy, by an application of Lemma 3.1.

Proposition 3.2. Let Assumptions (H1), (H2)(1), and (H2)(3) hold. Then

$$
E(t) \leq M_{1}(E(0))\left(1+\int_{0}^{t} \gamma(\tau) \mathrm{d} \tau\right)^{-\frac{p+2}{p}}, \quad \forall t \geq 0,
$$

for some positive function $M_{1}$ on $R_{+}$that is bounded on bounded sets.

Proof. It suffices to show the estimate for strong solutions, by a density argument.

First we consider the case $\alpha_{1}=\alpha_{2}=\alpha$ and take $\eta(t)=R_{1}(1+t)^{-\alpha}$. By (1.2) we see that

$$
\left|\frac{\eta^{\prime}(t)}{\eta(t) \gamma(t)}\right| \leq \frac{\alpha}{R_{1}}(1+t)^{\alpha-1}
$$

Thus, for $V_{1}$ and $V_{2}$ in (3.2) we have

$$
V_{1}(t) \leq C_{4}, \quad V_{2}(t) \leq C_{4}, \quad t \geq 0,
$$

with a constant $C_{4}>0$. Accordingly,

$$
E_{\varepsilon, \gamma}^{\prime}(t) \leq-\gamma(t)\left(\frac{1}{2}-\varepsilon \tilde{C} C_{4}\right)\left\|u^{\prime}\right\|^{2}+\varepsilon R_{1}\left(\varepsilon \tilde{C} C_{4}-\tilde{c}\right)(1+t)^{-\alpha}[E(t)]^{1+r}
$$

by virtue of Lemma 3.1. On the other hand, using the Cauchy-Schwarz inequality, (1.2) and (3.1), we obtain

$$
\begin{aligned}
\varepsilon R_{1}(1+t)^{-\alpha}[E(t)]^{r}\left|\left\langle u^{\prime}, u\right\rangle\right| & \leq \varepsilon R_{1}[E(t)]^{r}\left\|u^{\prime}\right\|\|u\| \\
& \leq C_{5} \varepsilon[E(t)]^{r+\frac{1}{2}+\frac{1}{p+2}}=C_{5} \varepsilon[E(t)]^{1+\frac{p}{2(p+2)}} \\
& \leq C_{6} \varepsilon E(t) .
\end{aligned}
$$

Take $\varepsilon=\tilde{c}\left(2 \tilde{C} C_{4}+2 C_{6}+\tilde{c}\right)^{-1}$. Then $\varepsilon \in(0,1)$, and

$$
\varepsilon \tilde{C} C_{4} \leq c / 2<1 / 2, \quad C_{6} \varepsilon \leq 1 / 2 .
$$

Thus, it follows from (3.8) and (3.7) that

$$
\frac{1}{2} E(t) \leq E_{\varepsilon, \gamma}(t) \leq 2 E(t), \quad \forall t \geq 0
$$

and

$$
E_{\varepsilon, \gamma}^{\prime}(t) \leq-\frac{1}{2} \tilde{c} R_{1} \varepsilon(1+t)^{-\alpha}[E(t)]^{1+r} \leq-\left(\frac{1}{2}\right)^{2+r} \tilde{c} R_{1} \varepsilon(1+t)^{-\alpha}\left[E_{\varepsilon, \gamma}(t)\right]^{1+r}
$$


Therefore,

$$
E(t) \leq 2 E_{\varepsilon, \gamma}(t) \leq C_{7}\left(1+\int_{0}^{t}(1+\tau)^{-\alpha} \mathrm{d} \tau\right)^{-\frac{1}{r}} \leq C_{7}\left(1+\int_{0}^{t} \gamma(\tau) \mathrm{d} \tau\right)^{-\frac{1}{r}}
$$

When $\alpha_{1} \neq \alpha_{2}$, by (H2)(3) we derive

$$
\left|\gamma^{\prime}(t)\right| \leq C_{0} \gamma^{2}(t), \quad t \geq 0
$$

Therefore, taking $\eta(t)=\gamma(t)$, we see that

$$
\left|\frac{\eta^{\prime}(t)}{\eta(t) \gamma(t)}\right|=\left|\frac{\gamma^{\prime}(t)}{\gamma(t)^{2}}\right|
$$

is also bounded. Then, arguing similarly as in the paragraph above, and using (1.2) whenever in need, we still obtain the energy estimate as in (3.9).

The proof is complete.

Finally, we make an improvement over the upper estimate of energy in Proposition 3.2, which will play a key role for the case $\alpha_{1}>1 / 2$, by following the way as in Section 2 of [31] (see also [7], Sect. 3.1) with effective adaptations for the present situation.

Proposition 3.3. Suppose $1 / 2<\alpha_{1}<1$. Let Assumptions (H1) and (H2)(1)-(4) hold. Then

$$
E(t) \leq M_{2}(E(0))\left(\frac{1}{1+t}\right)^{\left(\alpha_{1}+\alpha_{2}\right) \cdot \frac{p+2}{p}}, \quad \forall t \geq 0,
$$

for some positive function $M_{2}$ that are bounded on bounded sets.

Proof. First we write

$$
k_{0}:=\left(1-\alpha_{1}\right) \cdot \frac{p+2}{p}, \quad k_{*}:=\left(\alpha_{1}+\alpha_{2}\right) \frac{p+2}{p} .
$$

It is clear that $k_{0}<k_{*}$. Applying Proposition 3.2 enables us to obtain

$$
E(t) \leq C_{8}\left(\frac{1}{1+\int_{0}^{t} \gamma(\tau) \mathrm{d} \tau}\right)^{\frac{p+2}{p}} \leq C_{8}(1+t)^{-k_{0}}, \quad \forall t \geq 0
$$

by (1.2).

Consider the function $q(t):=\frac{1}{2}\|u(t)\|^{2}$. Using (H2)(1) and (1.1), we have

$$
\begin{aligned}
q^{\prime \prime}(t)+\gamma(t) q^{\prime}(t) & =\left\|u^{\prime}\right\|^{2}-\left\|A^{1 / 2} u\right\|^{2}-\langle f(u), u\rangle \\
& \leq\left\|u^{\prime}\right\|^{2}-\min \{2, c\}\left(\frac{1}{2}\left\|A^{1 / 2} u\right\|^{2}+F(u)\right) \\
& \leq 2\left\|u^{\prime}\right\|^{2}-\min \{2, c\} E(t) .
\end{aligned}
$$


Therefore,

$$
\min \{2, c\} \int_{0}^{T} \lambda_{k}(t) E(t) \mathrm{d} t \leq 2 \int_{0}^{T} \lambda_{k}(t)\left\|u^{\prime}(t)\right\|^{2} \mathrm{~d} t-\int_{0}^{T} \lambda_{k}(t) q^{\prime \prime}(t) \mathrm{d} t-\int_{0}^{T} \lambda_{k}(t) \gamma(t) q^{\prime}(t) \mathrm{d} t,
$$

where

$$
T>0, \quad \lambda_{k}(t):=(1+t)^{k} \text { with } k \in\left[k_{0}-1, k_{*}\right] .
$$

Integrating by parts yields that

$$
\begin{aligned}
\min \{2, c\} \int_{0}^{T} \lambda_{k}(t) E(t) \mathrm{d} t \leq & 2 \int_{0}^{T} \lambda_{k}\left\|u^{\prime}(t)\right\|^{2} \mathrm{~d} t-\lambda_{k}(T) q^{\prime}(T)+\left[\lambda_{k}^{\prime}-\left(\gamma \lambda_{k}\right)\right](T) q(T) \\
& +\int_{0}^{T}\left[\left(\lambda_{k} \gamma\right)^{\prime}-\lambda_{k}^{\prime \prime}\right](t) q(t) \mathrm{d} t+q^{\prime}(0)+(\gamma(0)-k) q(0) .
\end{aligned}
$$

Notice

$$
\lambda_{k}^{\prime}(t)=k(1+t)^{k-1} \quad \text { and } \quad k \leq k_{*}<\frac{2 p+4}{p} .
$$

We see

$$
\lambda_{k}^{\prime}(T)-\left(\gamma \lambda_{k}\right)(T) \leq-\frac{1}{2}\left(\gamma \lambda_{k}\right)(T), \quad \forall T \geq T_{0},
$$

where

$$
T_{0}:=\left(\frac{4 p+8}{p R_{1}}\right)^{1 /\left(1-\alpha_{1}\right)}
$$

Also,

$$
\left|q^{\prime}(t)\right| \leq\left\|u^{\prime}(t)\right\|\|u(t)\| \leq 2 \sqrt{E(t)} \sqrt{q(t)} .
$$

Therefore,

$$
\begin{aligned}
& -\lambda_{k}(T) q^{\prime}(T)+\left[\lambda_{k}^{\prime}-\left(\gamma \lambda_{k}\right)\right](T) q(T) \\
\leq & {\left[2\left(\lambda_{k} \sqrt{E}\right)(T)\right] \sqrt{q(T)}-\left[\frac{1}{2}\left(\lambda_{k} \gamma\right)(T)\right](\sqrt{q(T)})^{2} } \\
\leq & \frac{\left[2\left(\lambda_{k} \sqrt{E}\right)(T)\right]^{2}}{2\left(\lambda_{k} \gamma\right)(T)} \quad(\text { the maximum of the above formal function of } \sqrt{q(T)}) \\
\leq & \frac{2}{R_{1}} \lambda_{k+\alpha_{1}}(T) E(T) \quad(\text { by }(1.2)), \quad \forall T \geq T_{0} .
\end{aligned}
$$

Accordingly, we infer, for $T \geq T_{0}$,

$$
\int_{0}^{T} \lambda_{k}(t) E(t) \mathrm{d} t \leq \bar{C}_{0} \int_{0}^{T} \lambda_{k}(t)\left\|u^{\prime}(t)\right\|^{2} \mathrm{~d} t+\bar{C}_{0} \lambda_{k+\alpha_{1}}(T) E(T)+\bar{C}_{0} \int_{0}^{T}\left[\left(\lambda_{k} \gamma\right)^{\prime}-\lambda_{k}^{\prime \prime}\right](t) q(t) \mathrm{d} t+C_{9}
$$




$$
:=\bar{C}_{0}\left(J_{1}+J_{2}+J_{3}\right)+C_{9}, \quad \forall T \geq T_{0},
$$

with constants $\bar{C}_{0}, C_{9}>0$.

From (1.2) and (3.10) it follows that

$$
\begin{aligned}
J_{1} & =\int_{0}^{T} \frac{\lambda_{k}}{\gamma(t)} \gamma(t)\left\|u^{\prime}(t)\right\|^{2} \mathrm{~d} t \leq \frac{1}{R_{1}} \int_{0}^{T} \lambda_{k+\alpha_{1}}\left(-E^{\prime}\right) \mathrm{d} t \\
& \leq-\frac{1}{R_{1}} \lambda_{k+\alpha_{1}}(T) E(T)+\frac{E(0)}{R_{1}}+\frac{k+\alpha_{1}}{R_{1}} \int_{0}^{T} \lambda_{k+\alpha_{1}-1}(t) E(t) \mathrm{d} t \\
& \leq \frac{E(0)}{R_{1}}+\frac{C_{8}\left(k+\alpha_{1}\right)}{R_{1}} \int_{0}^{T} \lambda_{k+\alpha_{1}-1-k_{0}}(t) \mathrm{d} t .
\end{aligned}
$$

Also using (3.10) gives

$$
J_{2} \leq \frac{2 C_{8}}{R_{1}} \lambda_{k+\alpha_{1}-k_{0}}(T)
$$

As for $J_{3}$, we observe

$$
\left(\lambda_{k} \gamma\right)^{\prime}(t)=\lambda_{k}^{\prime}(t) \gamma(t)+\lambda_{k}(t) \gamma^{\prime}(t) \leq(k+C) R_{2}(1+t)^{k-1}(1+t)^{-\alpha_{2}},
$$

by $(1.2)$ and $(\mathrm{H} 2)(4)$,

$$
\lambda_{k}^{\prime \prime}(t)=k(k-1)(1+t)^{k-2},
$$

and

$$
q(t)=\frac{1}{2}\|u(t)\|^{2} \leq C_{10}[E(t)]^{\frac{2}{p+2}} \leq C_{11}(1+t)^{-\frac{2 k_{0}}{p+2}},
$$

by (3.10) again. Accordingly,

$$
J_{3} \leq C_{12} \int_{0}^{T}(1+t)^{k-\alpha_{2}-1-\frac{2 k_{0}}{p+2}} \mathrm{~d} t
$$

Take $k=k_{0}-\alpha_{1}-\epsilon$, where $\epsilon$ is a fixed positive number satisfying $1-\alpha_{1}-\epsilon>0$. Noticing

$$
\tilde{k}-\alpha_{1} \leq \frac{2 \tilde{k}}{p+2}+\alpha_{2}, \quad \text { whenever } \tilde{k} \in\left[k_{0}, k_{*}\right]
$$

we see that

$$
\left\{\begin{array}{l}
k<k_{0}-\alpha_{1}, \\
k<\frac{2 k_{0}}{p+2}+\alpha_{2} .
\end{array}\right.
$$

Accordingly, combining (3.11) with (3.12)-(3.14) yields that

$$
\int_{0}^{+\infty} \lambda_{k} E(t) \mathrm{d} t \leq C_{13} .
$$


Since $E(t)$ is decreasing, we see

$$
E(t) \int_{t / 2}^{t} \lambda_{k}(s) \mathrm{d} s \leq \int_{t / 2}^{t} \lambda_{k} E(s) \mathrm{d} s
$$

Hence,

$$
E(t) \leq C_{14}\left(\frac{1}{1+t}\right)^{k+1}=C_{14}\left(\frac{1}{1+t}\right)^{k_{1}}, \quad t \geq 0
$$

where

$$
k_{1}:=k_{0}+1-\alpha_{1}-\epsilon
$$

If $k_{1} \geq k_{*}$, then we have obtained the required energy estimate. If not, then

$$
E(t) \leq C_{15}\left(\frac{1}{1+t}\right)^{k_{2}}, \quad t \geq 0
$$

where

$$
k_{2}:=k_{1}+1-\alpha_{1}-\epsilon=k_{0}+2\left(1-\alpha_{1}-\epsilon\right)
$$

this is because (3.12)-(3.14), with $k_{0}$ replaced by $k_{1}$, are satisfied, by using (3.15) (instead of (3.10)). Thus, proceeding like this and denoting by $n$ the positive integer satisfying

$$
\left\{\begin{array}{l}
k_{0}+n\left(1-\alpha_{1}-\epsilon\right)<k_{*}, \\
k_{n}:=k_{0}+(n+1)\left(1-\alpha_{1}-\epsilon\right) \geq k_{*},
\end{array}\right.
$$

we obtain

$$
E(t) \leq C_{16}\left(\frac{1}{1+t}\right)^{k_{n}} \leq C_{17}\left(\frac{1}{1+t}\right)^{k_{*}}, \quad t \geq 0
$$

This ends the proof.

\section{Proofs of the TheOREMS}

Throughout the section, $\bar{C}_{1}, \bar{C}_{2}, \ldots, \bar{C}_{15}$ denote positive constants depending on the values of $E(0)$ and being bounded on bounded sets of the values, and they may be different at different positions.

\subsection{Proof of Theorem 2.2}

It suffices to deal with the strong solution case.

We divide the proof into three steps.

Step 1. Obtain a better estimate of energy for case $\alpha_{1} \leq 1 / 2$. 
Take $\eta(t)=1$ in Lemma 3.1. Then $V_{2}(t)=\gamma(t)$ is bounded. For $V_{1}(t)$ we exploit Proposition 3.2 to deduce that

$$
E(t)^{r} \leq \bar{C}_{1}\left(1+\int_{0}^{t} \gamma(\tau) \mathrm{d} \tau\right)^{-\frac{p+2}{p} \cdot r} \leq \bar{C}_{1}\left(\frac{1}{1+t}\right)^{1-\alpha_{1}} \leq \bar{C}_{1}\left(\frac{1}{1+t}\right)^{\alpha_{1}} \leq \bar{C}_{1} \gamma(t),
$$

since $\alpha_{1} \leq 1 / 2$. Accordingly,

$$
V_{1}(t) \leq \bar{C}_{2} \gamma(t)
$$

Thus, similarly as in the proof of Proposition 3.2, we can let $\varepsilon$ small enough such that

$$
\frac{1}{2} E(t) \leq E_{\varepsilon, 1}(t) \leq 2 E(t), \quad \forall t \geq 0
$$

and

$$
E_{\varepsilon, 1}^{\prime}(t) \leq-\frac{1}{2} \tilde{c} \varepsilon[E(t)]^{1+r}
$$

This gives that

$$
E(t) \leq \bar{C}_{3}(1+t)^{-\frac{1}{r}}=\bar{C}_{3}(1+t)^{-\frac{p+2}{p}}
$$

Step 2. Obtain a better estimate of energy for case $\alpha_{1}>1 / 2$.

Take

$$
\eta(t)=(1+t)^{2 \alpha_{1}-1}
$$

in Lemma 3.1. Making use of Proposition 3.3 we infer that

$$
\eta(t)[E(t)]^{\frac{p}{2 p+4}} \leq \bar{C}_{4}(1+t)^{2 \alpha_{1}-1}(1+t)^{-\frac{\alpha_{1}+\alpha_{2}}{2}} \leq \bar{C}_{4}(1+t)^{2 \alpha_{1}-1}(1+t)^{-\alpha_{2}} \leq \bar{C}_{4},
$$

by $(\mathrm{H} 2)(5)$. This yields that

$$
\varepsilon \eta(t)[E(t)]^{r}\left|\left\langle u^{\prime}, u\right\rangle\right| \leq \varepsilon \eta(t)[E(t)]^{r}\left\|u^{\prime}\right\|\|u\| \leq \bar{C}_{5} \varepsilon \eta(t)[E(t)]^{1+\frac{p}{2 p+4}} \leq \bar{C}_{5} \varepsilon E(t),
$$

which implies that

$$
\frac{1}{2} E(t) \leq E_{\varepsilon, \eta}(t) \leq 2 E(t), \quad \forall t \geq 0
$$

whenever $\varepsilon$ is small enough.

Moreover, using Proposition 3.3 again, we obtain

$$
\eta(t)[E(t)]^{r} \leq \bar{C}_{6}(1+t)^{2 \alpha_{1}-1}(1+t)^{-\alpha_{1}-\alpha_{2}}=\bar{C}_{6}(1+t)^{2 \alpha_{1}-1-\alpha_{2}}(1+t)^{-\alpha_{1}} \leq \bar{C}_{6} \gamma(t),
$$

due to $(\mathrm{H} 2)(5)$ and (1.2). Furthermore, we have

$$
\gamma(t) \eta(t) \leq R_{2}(1+t)^{-\alpha_{2}}(1+t)^{2 \alpha_{1}-1} \leq R_{2},
$$


and

$$
\eta^{\prime}(t)=\left(2 \alpha_{1}-1\right)(1+t)^{2 \alpha_{1}-2} \leq \bar{C}_{7} \gamma(t) \eta(t)
$$

by (1.2). These estimates combined with (4.2) indicate that $V_{2}(t)$ is bounded, and

$$
V_{1}(t) \eta(t) \leq \bar{C}_{8} \gamma(t) .
$$

Therefore, there exists a sufficiently small $\varepsilon$ such that (4.3) is satisfied, and

$$
E_{\varepsilon, \eta}^{\prime}(t) \leq-\frac{1}{2} \tilde{c} \varepsilon \eta(t)[E(t)]^{1+r}
$$

Consequently,

$$
E_{\varepsilon, \eta}(t) \leq \bar{C}_{9}\left(1+\int_{0}^{t} \eta(\tau) \mathrm{d} \tau\right)^{-1 / r} .
$$

So

$$
E(t) \leq \bar{C}_{10}(1+t)^{-2 \alpha_{1} / r}=\bar{C}_{10}(1+t)^{-2 \alpha_{1} \cdot \frac{p+2}{p}} .
$$

Step 3. Achieve the desired estimate of energy.

We take

$$
\eta(t)=\eta_{0}(t):=\left\{\begin{array}{lll}
(1+t)^{\alpha}, & \text { if } & \alpha_{1}=\alpha_{2}=\alpha, \\
\gamma(t)^{-1}, & \text { if } & \alpha_{1} \neq \alpha_{2}
\end{array}\right.
$$

in Lemma 3.1. The boundedness of $V_{2}(t)$ is easy to see by (H2)(3).

Employing (4.1) and (4.4), we obtain

$$
[E(t)]^{r} \leq \bar{C}_{11}(1+t)^{-2 \alpha_{1}} \leq \bar{C}_{11} \gamma(t)^{2}
$$

by (1.2). This yields that

$$
[E(t)]^{\frac{p}{2 p+4}}=[E(t)]^{r / 2} \leq \bar{C}_{12} \gamma(t) .
$$

Hence

$$
V_{1}(t) \eta_{0}(t)=\left([E(t)]^{\frac{p}{2 p+4}} \gamma(t)+[E(t)]^{r}\right) \eta_{0}(t) \leq \bar{C}_{13} \gamma(t) .
$$

Moreover,

$$
\varepsilon \eta_{0}(t)[E(t)]^{r}\left|\left\langle u^{\prime}, u\right\rangle\right| \leq \bar{C}_{14} \varepsilon \eta_{0}(t)[E(t)]^{1+\frac{p}{2(p+2)}} \leq \bar{C}_{14} \varepsilon E(t) .
$$

Accordingly, choosing $\varepsilon$ small enough we deduce that

$$
\frac{1}{2} E(t) \leq E_{\varepsilon, \eta_{0}}(t) \leq 2 E(t), \quad \forall t \geq 0,
$$




$$
E_{\varepsilon, \eta_{0}}^{\prime}(t) \leq-\frac{1}{2} \tilde{c} \varepsilon \eta_{0}(t)[E(t)]^{1+r}
$$

Therefore,

$$
E(t) \leq \bar{C}_{15}\left(1+\int_{0}^{t} \eta_{0}(\tau) \mathrm{d} \tau\right)^{-1 / r} \leq \bar{C}_{15}\left(1+\int_{0}^{t} \gamma(\tau)^{-1} \mathrm{~d} \tau\right)^{-1 / r}
$$

\subsection{Proof of Theorem 2.3}

We follow the strategy in Section 3.4 of [18] (for the case of $\gamma(t)=1$ ) to prove the theorem. It is worth noting that exploitations of the (already obtained) fine upper bound estimates will play an important role.

Let $u(t)$ be a mild solution of $(1.1)$ with $u_{0} \neq 0$. Set

$$
\gamma_{1}=\frac{R_{2}^{2}\left(C_{0}^{2}+1\right)}{\left\|u_{0}\right\|^{2 p+2}}\left(\left\|u_{1}\right\|^{2}+\left\|A^{1 / 2} u_{0}\right\|^{2}\right)+\frac{129 C_{0}^{2} M^{2}}{\delta^{2}}
$$

where $R_{2}$ is as in (1.2), $C_{0}$ as in (H3)(3), $M$ as in (H3)(2), and $\delta \in(0,1 / 2)$ is a constant that will be determined later; set

$$
G(t):=\frac{\left\|u^{\prime}(t)\right\|^{2}+\left\|A^{1 / 2} u(t)\right\|^{2}}{2\|u(t)\|^{2 p+2}}
$$

We will show the existence of a nonempty open set $S \subset\left(H_{1} \backslash\{0\}\right) \times H$ such that for $\left(u_{0}, u_{1}\right) \in S$, the solution $u(t)$ of problem (1.1) satisfies the property: given $T>0$, one has

$$
u(T) \neq 0 \quad \text { and } \quad G(T)<\frac{2 \gamma_{1}}{\gamma^{2}(T)}
$$

whenever

$$
u(t) \neq 0 \quad \text { and } \quad G(t) \leq \frac{2 \gamma_{1}}{\gamma^{2}(t)} \quad \text { for all } t \in[0, T)
$$

Next, we assume (4.8). We will find such a set $S$, and with it prove (4.7) (for strong solutions), by seven steps.

Step 1. Construct a small perturbation of $G(t)$.

Denoting by $Q$ the orthogonal projection from $H$ to $(\operatorname{ker} A)^{\perp}$, we set

$$
\hat{G}(t)=G(t)+\delta \gamma(t) \frac{\left\langle u^{\prime}(t), Q u(t)\right\rangle}{\|u(t)\|^{2 p+2}}, \quad t \in[0, T),
$$

for the case of $\alpha_{1} \neq \alpha_{2}$; replace $\gamma(t)$ by $(1+t)^{-\alpha}$ in (4.9) for the case of $\alpha_{1}=\alpha_{2}=\alpha$. Below we only address the former case (the latter case can be dealt with similarly). We have

$$
\begin{aligned}
\hat{G}^{\prime}(t)= & -\gamma(t) \frac{\left\|u^{\prime}\right\|^{2}}{\|u\|^{2 p+2}}-\delta \gamma(t) \frac{\left\|A^{1 / 2} u\right\|^{2}}{\|u\|^{2 p+2}}+\delta \gamma(t) \frac{\left\|Q u^{\prime}\right\|^{2}-\gamma(t)\left\langle u^{\prime}, Q u\right\rangle}{\|u(t)\|^{2 p+2}} \\
& -\frac{\left\langle f(u), u^{\prime}+\delta \gamma(t) Q u\right\rangle}{\|u\|^{2 p+2}}-(2 p+2) \frac{\left\langle u^{\prime}, u\right\rangle}{\|u\|^{2}} \cdot \hat{G}(t)+\delta \gamma^{\prime}(t) \frac{\left\langle u^{\prime}, Q u\right\rangle}{\|u\|^{2 p+2}} \\
& =: K_{1}+K_{2}+K_{3}+K_{4}+K_{5}+K_{6}, \quad t \in[0, T) .
\end{aligned}
$$


Step 2. Estimate $K_{3}$ and $K_{6}$.

It follows from (1.2) and (H3)(1) that

$$
\gamma(t)\left\langle u^{\prime}, Q u\right\rangle \leq \frac{R_{2}}{\sqrt{\zeta}}\left\|u^{\prime}\right\| \sqrt{\zeta}\|Q u\| \leq \frac{R_{2}}{\sqrt{\zeta}}\left\|u^{\prime}\right\|\left\|A^{1 / 2} u\right\| \leq \frac{2 R_{2}^{2}}{\zeta}\left\|u^{\prime}\right\|^{2}+\frac{1}{8}\left\|A^{1 / 2} u\right\|^{2},
$$

noting that $\left\|A^{1 / 2} Q u\right\|=\left\|A^{1 / 2} u\right\|$. Hence, we have

$$
K_{3} \leq \tilde{D}_{1} \delta \gamma(t) \frac{\left\|u^{\prime}\right\|^{2}}{\|u\|^{2 p+2}}+\frac{1}{8} \delta \gamma(t) \frac{\left\|A^{1 / 2} u\right\|^{2}}{\|u\|^{2 p+2}} \leq \tilde{D}_{1} \delta \gamma(t) \frac{\left\|u^{\prime}\right\|^{2}}{\|u\|^{2 p+2}}+\frac{1}{4} \delta \gamma(t) G(t), \quad t \in[0, T),
$$

where $\tilde{D}_{1}:=\left(2 R_{2}^{2}+\zeta\right) / \zeta$.

As for $K_{6}$, we use (H2)(3) to get

$$
\left|\gamma^{\prime}(t)\right| \leq C \gamma^{2}(t) \leq C R_{2} \gamma(t), \quad \forall t \geq 0
$$

Also,

$$
\left|\left\langle u^{\prime}, Q u\right\rangle\right| \leq \frac{1}{\sqrt{\zeta}}\left\|u^{\prime}\right\| \sqrt{\zeta}\|Q u\| \leq \frac{1}{\sqrt{\zeta}}\left\|u^{\prime}\right\|\left\|A^{1 / 2} u\right\| \leq \frac{2 C R_{2}}{\zeta}\left\|u^{\prime}\right\|^{2}+\frac{1}{8 C R_{2}}\left\|A^{1 / 2} u\right\|^{2} .
$$

Accordingly,

$$
K_{6} \leq \tilde{D}_{2} \delta \gamma(t) \frac{\left\|u^{\prime}\right\|^{2}}{\|u\|^{2 p+2}}+\frac{\delta \gamma(t)}{4} G(t), \quad t \in[0, T),
$$

where $\tilde{D}_{2}:=2 C^{2} R_{2}^{2} / \zeta$.

Step 3. Estimate $K_{4}$ and $K_{5}$ in the case $\alpha<1 / 2$.

From now on, we assume

$$
E(0) \leq 1, \text { and }\left(\sup _{s \in[0,1]} \bar{C}(s)+2\right)[E(0)]^{\frac{2}{p+2}} \leq \xi^{2},
$$

where $\bar{C}$ and $\xi$ are, respectively, as in (3.1) and (H3)(2). Then

$$
\begin{aligned}
\|u(t)\|_{H_{1}}^{2} & =\|u(t)\|^{2}+\left\|A^{1 / 2} u(t)\right\|^{2} \leq\left(\sup _{s \in[0,1]} \bar{C}(s)\right)[E(t)]^{\frac{2}{p+2}}+2 E(t) \\
& \leq\left(\sup _{s \in[0,1]} \bar{C}(s)+2\right)[E(0)]^{\frac{2}{p+2}} \leq \xi^{2} .
\end{aligned}
$$

Thus, in view of (2.1), (4.1), (3.1), (1.2) and (H3)(2), we deduce that

$$
\begin{aligned}
\frac{\|f(u)\|}{\|u\|^{p+1}} & \leq M\left(\frac{\|u\|^{p+1}+\left\|A^{1 / 2} u\right\|^{p+1}}{\|u\|^{p+1}}\right) \\
& \leq M\left(1+\frac{\left\|A^{1 / 2} u\right\|}{\|u\|^{p+1}} \cdot[2 E(t)]^{p / 2}\right) \\
& \leq M\left(1+\sqrt{2 G(t)} \cdot[2 E(t)]^{p / 4}[2 E(0)]^{p / 4}\right)
\end{aligned}
$$




$$
\begin{aligned}
& \leq M\left(1+D_{1} \sqrt{G(t)} \cdot(1+t)^{-\frac{p+2}{4}}[E(0)]^{p / 4}\right) \\
& \leq M\left(1+D_{2}[E(0)]^{p / 4} \gamma(t) \sqrt{G(t)}\right), \quad t \in[0, T),
\end{aligned}
$$

noting

$$
\frac{p+2}{4}>\frac{1}{2}>\alpha_{1}
$$

Here, $D_{1}, D_{2}$ are positive constants independent of initial data (because of $E(0) \leq 1$ ). Also, we have

$$
\delta \gamma(t)\|Q u\| \leq \frac{\delta R_{2}}{\sqrt{\zeta}}\left\|A^{1 / 2} u\right\|,
$$

by $(\mathrm{H} 3)(1)$. Hence,

$$
\frac{\left\|u_{t}\right\|+\delta \gamma(t)\|Q u\|}{\|u\|^{p+1}} \leq \frac{\left\|u_{t}\right\|+R_{2} / \sqrt{\zeta}\left\|A^{1 / 2} u\right\|}{\|u\|^{p+1}} \leq D_{3} \sqrt{G(t)}, \quad t \in[0, T),
$$

where $D_{3}:=2\left(1+R_{2} / \sqrt{\zeta}\right)$. Thus,

$$
K_{4} \leq \frac{f(u)}{\|u\|^{p+1}} \cdot \frac{\left\|u_{t}\right\|+\delta \gamma(t)\|Q u\|}{\|u\|^{p+1}} \leq D_{3} M \sqrt{G(t)}+M D_{2} D_{3}[E(0)]^{p / 4} \gamma(t) G(t) .
$$

So

$$
K_{4} \leq \frac{4 M^{2}}{\delta \gamma(t)}+\frac{\delta \gamma(t)}{4} G(t)+\tilde{D}_{3}[E(0)]^{p / 4} \gamma(t) G(t), \quad t \in[0, T)
$$

where $\tilde{D}_{3}$ is a positive constant independent of initial data.

For $K_{5}$, we note that

$$
\exists \theta>0 \text { such that } \alpha_{1}+\theta=1 / 2,
$$

since $\alpha_{1}<1 / 2$. From the definition of $G(t),(3.1),(4.8)$ and (4.1), it follows that for $t \in[0, T)$,

$$
\begin{aligned}
\left|\frac{\left\langle u^{\prime}, u\right\rangle}{\|u\|^{2}}\right| & \leq \frac{\left\|u^{\prime}\right\|}{\|u\|^{1+p}} \cdot\|u\|^{p} \\
& \leq \sqrt{2 G(t)} \cdot\|u\|^{2 p \alpha_{1}}\|u\|^{2 p \theta} \\
& \leq \frac{\sqrt{\gamma_{1}}}{\gamma(t)} \cdot D_{4}(1+t)^{-2 \alpha_{1}}[E(0)]^{\frac{2 p \theta}{p+2}} \\
& \leq D_{5} \sqrt{\gamma_{1}} \gamma(t)[E(0)]^{\frac{2 p \theta}{p+2}}
\end{aligned}
$$

with positive constants $D_{4}, D_{5}$ independent of initial data. Therefore

$$
K_{5} \leq \tilde{D}_{4}[E(0)]^{\frac{p \theta^{\prime}}{p+2}} \sqrt{\gamma_{1}} \gamma(t) \cdot \hat{G}(t), \quad t \in[0, T),
$$

for some $\theta^{\prime}>0$, and some positive constant $\tilde{D}_{4}$ independent of initial data. 
Step 4. Estimate $K_{4}$ and $K_{5}$ in the case $\alpha \geq 1 / 2$.

In view of $(2.1),(4.4)$ and $(\mathrm{H} 3)(2)$, we obtain

$$
\begin{aligned}
\frac{\|f(u)\|}{\|u\|^{p+1}} & \leq M\left(1+\frac{\left\|A^{1 / 2} u\right\|}{\|u\|^{p+1}} \cdot[2 E(t)]^{p / 4}[2 E(0)]^{p / 4}\right) \\
& \leq M\left(1+D_{6} \sqrt{G(t)} \cdot(1+t)^{-\alpha_{1} \frac{p+2}{2}}[E(0)]^{p / 4}\right) \\
& \left.\leq M\left(1+D_{7} E(0)\right]^{p / 4} \gamma(t) \sqrt{G(t)}\right), \quad t \in[0, T),
\end{aligned}
$$

with positive constants $D_{6}, D_{7}$ independent of initial data. This means that the estimate (4.14) for $K_{4}$ holds too in this case.

By virtue of Theorem 2.2, we get

$$
\|u(t)\| \leq D_{8}\left(1+\int_{0}^{t} \gamma(\tau)^{-1} \mathrm{~d} \tau\right)^{-\frac{1}{p}} \leq D_{9}(1+t)^{-\frac{1+\alpha_{2}}{p}},
$$

where $D_{8}, D_{9}$ are positive constants independent of initial data. In addition,

$$
\exists \theta_{1} \in(0,1) \text { such that } 2 \alpha_{1}=\theta_{1}\left(1+\alpha_{2}\right)
$$

by $(\mathrm{H} 2)(5)$. Hence

$$
\begin{aligned}
\left|\frac{\left\langle u^{\prime}, u\right\rangle}{\|u\|^{2}}\right| & \leq \sqrt{2 G(t)} \cdot\|u\|^{p \theta_{1}}\|u\|^{\left(1-\theta_{1}\right) p} \\
& \leq \frac{\sqrt{\gamma_{1}}}{\gamma(t)} \cdot D_{10}(1+t)^{-\theta_{1}\left(1+\alpha_{2}\right)} \cdot[E(0)]^{\left(1-\theta_{1}\right) \frac{p}{p+2}} \\
& =\frac{\sqrt{\gamma_{1}}}{\gamma(t)} \cdot D_{10}(1+t)^{-2 \alpha_{1}}[E(0)]^{\left(1-\theta_{1}\right) \frac{p}{p+2}} \\
& \leq D_{11} \sqrt{\gamma_{1}} \gamma(t)[E(0)]^{\frac{\left(1-\theta_{1}\right) p}{p+2}}, \quad t \in[0, T),
\end{aligned}
$$

where $D_{10}, D_{11}$ are positive constants independent of initial data. So the estimate (4.15) for $K_{5}$ holds too in this case.

Step 5. Estimate $\hat{G}^{\prime}$.

Plugging (4.11)-(4.15) into (4.10), we obtain

$$
\begin{aligned}
\hat{G}^{\prime}(t) \leq & -\left(1-\tilde{D}_{1} \delta-\tilde{D}_{2} \delta\right) \gamma(t) \frac{\left\|u^{\prime}\right\|^{2}}{\|u\|^{2 p+2}}-\delta \gamma(t) \frac{\left\|A^{1 / 2} u\right\|^{2}}{\|u\|^{2 p+2}}+\frac{4 M^{2}}{\delta \gamma(t)} \\
& +\left(3 \delta / 4+\tilde{D}_{3}[E(0)]^{p / 4}\right) \gamma(t) G(t)+\tilde{D}_{4} \sqrt{\gamma_{1}}[E(0)]^{\frac{p \theta^{\prime}}{p+2}} \cdot \gamma(t) \hat{G}(t), \quad t \in[0, T) .
\end{aligned}
$$

From (H3)(1) and (1.2), we know that

$$
\gamma(t) \frac{\left\langle u^{\prime}, Q u\right\rangle}{\|u\|^{2 p+2}} \leq 2\left(R_{2}^{2} \zeta^{-1}+1 / 4\right) G(t), \quad t \in[0, T) .
$$


Now, we choose $\delta \in(0,1 / 2)$ such that

$$
\left\{\begin{array}{l}
\left(R_{2}^{2} \zeta^{-1}+1 / 4\right) \delta \leq 1 / 10 \\
\left(\tilde{D}_{1}+\tilde{D}_{2}\right) \delta \leq 1 / 2
\end{array}\right.
$$

then, choose a set $S$ of initial data satisfying (4.13) and

$$
\left\{\begin{array}{l}
\tilde{D}_{3}[E(0)]^{p / 4} \leq \delta / 32 \\
\tilde{D}_{4} \sqrt{\gamma_{1}}[E(0)]^{\frac{p \theta^{\prime}}{p+2}} \leq \delta / 32 .
\end{array}\right.
$$

We here emphasize that $\tilde{D}_{3}, \tilde{D}_{4}$ are independent of all the initial data satisfying $E(0) \leq 1$. Thus,

$$
\frac{5}{6} G(t) \leq \hat{G}(t) \leq 2 G(t), \quad t \in[0, T)
$$

and

$$
\begin{aligned}
\hat{G}^{\prime}(t) & \leq-\delta \gamma(t)\left(\frac{\left\|u^{\prime}\right\|^{2}}{\|u\|^{2 p+2}}+\frac{\left\|A^{1 / 2} u\right\|^{2}}{\|u\|^{2 p+2}}\right)+\frac{4 M^{2}}{\delta \gamma(t)}+\frac{25}{32} \delta \gamma(t) G(t)+\frac{1}{32} \delta \gamma(t) \hat{G}(t) \\
& \leq-\delta \gamma(t) \hat{G}(t)+\frac{4 M^{2}}{\delta \gamma(t)}+\frac{15}{16} \delta \gamma(t) \hat{G}(t)+\frac{1}{32} \delta \gamma(t) \hat{G}(t), \quad t \in[0, T),
\end{aligned}
$$

by noting

$$
\begin{aligned}
& -\left(1-\tilde{D}_{1} \delta-\tilde{D}_{2} \delta\right) \gamma(t) \frac{\left\|u^{\prime}\right\|^{2}}{\|u\|^{2 p+2}}-\delta \gamma(t) \frac{\left\|A^{1 / 2} u\right\|^{2}}{\|u\|^{2 p+2}} \\
\leq & -(1-1 / 2) \gamma(t) \frac{\left\|u^{\prime}\right\|^{2}}{\|u\|^{2 p+2}}-\delta \gamma(t) \frac{\left\|A^{1 / 2} u\right\|^{2}}{\|u\|^{2 p+2}} \\
\leq & -\min \{1 / 2, \delta\} \gamma(t)\left(\frac{\left\|u^{\prime}\right\|^{2}}{\|u\|^{2 p+2}}+\frac{\left\|A^{1 / 2} u\right\|^{2}}{\|u\|^{2 p+2}}\right),
\end{aligned}
$$

and $\delta \in(0,1 / 2)$. Hence,

$$
\hat{G}^{\prime}(t) \leq-\frac{1}{32} \delta \gamma(t) \hat{G}(t)+\frac{4 M^{2}}{\delta \gamma(t)}, \quad t \in[0, T) .
$$

Step 6. Estimate $\hat{G}$.

Integrating (4.17) and setting

$$
H(t):=\exp \left(\frac{\delta}{32} \int_{0}^{t} \gamma(\tau) \mathrm{d} \tau\right), \quad t \geq 0,
$$

we deduce that

$$
\hat{G}(t) \leq\left(\hat{G}(0)+\frac{4 M^{2}}{\delta} \int_{0}^{t} \gamma(s)^{-1} H(s) \mathrm{d} s\right) H(t)^{-1}, \quad t \in[0, T) .
$$


By $(\mathrm{H} 3)(3)$, we have

$$
\gamma^{-1}(s) \leq C_{0}^{2} \frac{\gamma(s)}{\gamma^{2}(t)}, \quad \forall t \geq s>0
$$

Then

$$
\hat{G}(t) \leq \hat{G}(0) H(t)^{-1}+\frac{4 C_{0}^{2} M^{2}}{\delta \gamma^{2}(t)} \frac{\int_{0}^{t} \gamma(s) H(s) \mathrm{d} s}{H(t)}, \quad t \in[0, T) .
$$

From

$$
H^{\prime}(t)=\frac{\delta}{32} \gamma(t) \exp \left(\frac{\delta}{32} \int_{0}^{t} \gamma(\tau) \mathrm{d} \tau\right)=\frac{\delta}{32} \gamma(t) H(t)
$$

it follows that

$$
\int_{0}^{t} \gamma(s) H(s) \mathrm{d} s=\frac{32}{\delta}(H(t)-H(0)) \leq \frac{32}{\delta} H(t) .
$$

Therefore, noting $H(t) \geq 1$ for $t \in[0, T)$, we derive

$$
\hat{G}(t) \leq \hat{G}(0)+\frac{128 C_{0}^{2} M^{2}}{\delta^{2} \gamma^{2}(t)}, \quad t \in[0, T) .
$$

Step 7. Obtain (4.7).

Using (4.8), we have

$$
\left|\frac{\mathrm{d}}{\mathrm{d} t}\|u(t)\|^{2}\right|=2\left|\left\langle u^{\prime}(t), u(t)\right\rangle\right| \leq 2 \sqrt{2 G(t)} \cdot\|u\|^{2+p} \leq \frac{4 \sqrt{\gamma_{1}}}{\gamma(t)}\left(\|u(t)\|^{2}\right)^{1+\frac{p}{2}}, \quad t \in[0, T) .
$$

So we obtain

$$
\|u(t)\|^{2} \geq\left(2 p \sqrt{\gamma_{1}} \int_{0}^{t} \gamma(s)^{-1} \mathrm{~d} s+\left\|u_{0}\right\|^{-p}\right)^{-\frac{2}{p}}, \quad t \in[0, T) .
$$

Thus, we see that $u(T) \neq 0$.

Letting $t \rightarrow T^{-}$in (4.18) and using (H3)(3), we get

$$
\hat{G}(T) \leq\left(\frac{1}{C_{0}^{2}} \cdot C_{0}^{2} \hat{G}(0)+\frac{128 C_{0}^{2} M^{2}}{\delta^{2} \gamma^{2}(T)}\right) \leq\left(\frac{\gamma^{2}(0)}{\gamma^{2}(T)} \cdot C_{0}^{2} \hat{G}(0)+\frac{128 C_{0}^{2} M^{2}}{\delta^{2} \gamma^{2}(T)}\right) .
$$

So

$$
G(T) \leq 2 \hat{G}(T) \leq \frac{2}{\gamma^{2}(T)}\left(2 R_{2}^{2} C_{0}^{2} \cdot G(0)+\frac{128 C_{0}^{2} M^{2}}{\delta^{2}}\right)<\frac{2 \gamma_{1}}{\gamma^{2}(T)}
$$

Therefore, (4.7) is satisfied. 
Consequently, for any $\left(u_{0}, u_{1}\right) \in S$ (given by (4.13) and (4.16)) and any $T>0$, we have

$$
u(T) \neq 0 \quad \text { and } \quad G(T)<\frac{2 \gamma_{1}}{\gamma^{2}(T)}
$$

provided $u(t)$ is a strong solution, and

$$
u(t) \neq 0 \quad \text { and } \quad G(t) \leq \frac{2 \gamma_{1}}{\gamma^{2}(t)}, \quad \text { for all } t \in[0, T)
$$

This implies that

$$
\sup \{T>0:(4.19) \text { holds }\}=+\infty
$$

due to the continuity of $G$ and the fact that (4.19) is indeed satisfied for some $T>0$, as can be seen from the estimate

$$
\gamma^{2}(0) G(0) \leq R_{2}^{2} G(0)<\gamma_{1} / 2
$$

Therefore, we obtain

$$
u(t) \neq 0 \quad \text { and } \quad G(t) \leq \frac{2 \gamma_{1}}{\gamma^{2}(t)}, \quad \text { for all } t \geq 0
$$

Accordingly,

$$
\left|\frac{\mathrm{d}}{\mathrm{d} t}\|u(t)\|^{2}\right| \leq \frac{4 \sqrt{\gamma_{1}}}{\gamma(t)}\left(\|u(t)\|^{2}\right)^{1+\frac{p}{2}}, \quad t \geq 0
$$

for strong solutions (and so for mild solution as well). Hence,

$$
\|u(t)\| \geq\left(2 p \sqrt{\gamma_{1}} \int_{0}^{t} \gamma^{-1}(s) \mathrm{d} s+\left\|u_{0}\right\|^{-p}\right)^{-\frac{1}{p}}, \quad \text { for all } t \geq 0 .
$$

This combined with (3.1) gives (2.4). Thus, we complete the proof.

\section{Applications}

In this section, $\Omega \subset R^{n}$ is a bounded domain with smooth boundary $\partial \Omega, \nu$ is the unit outward normal on $\partial \Omega$, and $\gamma(t)$ is a decreasing function on $R^{+}$satisfying (1.2) with $\alpha_{1}=\alpha_{2}=\alpha$.

Example 5.1. We consider the following nonautonomous wave equation with the Neumann boundary condition:

$$
\left\{\begin{array}{l}
u_{t t}(t, x)-\Delta u(t, x)+\gamma(t) u_{t}(t, x)+\left(\int_{\Omega}|u(t, x)|^{2} \mathrm{~d} x\right)^{p / 2} u(t, x)=0, \quad \text { in }[0,+\infty) \times \Omega, \\
u(0, x)=u_{0}(x), u_{t}(0, x)=u_{1}(x), \quad x \in \Omega \\
\frac{\partial u(t, x)}{\partial \nu}=0, \quad \text { on } \partial \Omega \times(0, \infty),
\end{array}\right.
$$

where $p \geq 1$. 
Take $H=L^{2}(\Omega)$, and define operator $A$ by

$$
A v(x)=-\Delta v(x), \quad x \in \Omega \text { a.e. }
$$

with

$$
v \in D(A):=\left\{u \in H^{2}(\Omega) ; \quad \frac{\partial u}{\partial \nu}=0 \text { on } \partial \Omega\right\}
$$

It is known that $A$ is a self-adjoint nonnegative operator on $H, H_{1}:=D\left(A^{1 / 2}\right)=H^{1}(\Omega)$, and (H3)(1) holds.

Moreover, we set

$$
f(v)(x)=\left(\int_{\Omega}|v(x)|^{2} \mathrm{~d} x\right)^{p / 2} v(x)=\|v\|^{p} v(x), \quad v \in H_{1} .
$$

Then

$$
\|f(v)\|=\|v\|^{1+p}, \quad \forall v \in H_{1},
$$

and so (H3)(2) is satisfied. Besides (H1)(ii) and (H2)(1)-(2) are also satisfied (cf. [28], Exam. 4.2 for details). Therefore, applying Theorems 2.2 and 2.3 (seeing also Rem. 2.4 (1)), we obtain the following conclusions regarding the mild solution $u(t)$ and the energy

$$
E_{u}(t):=\frac{1}{2} \int_{\Omega}\left(\left|u_{t}\right|^{2}+|\nabla u|^{2}\right) \mathrm{d} x+\frac{1}{p+2}\left(\int_{\Omega}|u(t, x)|^{2} \mathrm{~d} x\right)^{\frac{p+2}{2}}
$$

of the problem (5.1).

(i) For some positive function $M_{1}$ on $R_{+}$that are bounded on bounded sets,

$$
E(t),\|u(t)\|^{p+2} \leq M_{1}(E(0))(1+t)^{-(1+\alpha)(p+2) / p}, \quad \forall t \geq 0
$$

(ii) there exists a nonempty open set $S \subset H_{1} \times H$ such that for some positive function $c_{0}$ depending on $\left\|u_{0}\right\|$, $\left\|A^{1 / 2} u_{0}\right\|$ and $\left\|u_{1}\right\|$

$$
E(t),\|u(t)\|^{p+2} \geq c_{0}(1+t)^{-(1+\alpha)(p+2) / p}, \quad \forall t \geq 0
$$

whenever $\left(u_{0}, u_{1}\right) \in S$,

Example 5.2. Consider the Dirichlet problem for a nonautonomous wave equation

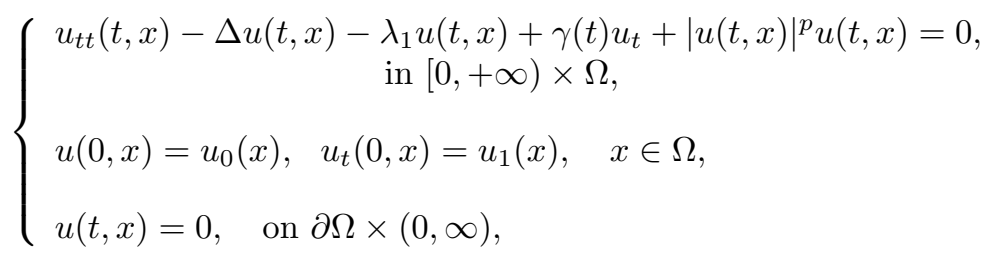


where $\lambda_{1}$ is the first eigenvalue of the negative Dirichlet-Laplacian on $\Omega, p>0$ and

$$
p \leq 2 /(n-2) \text { if } n>2 \text {. }
$$

Take $H=L^{2}(\Omega)$, and define operator $A$ by

$$
A v(x)=-\Delta v(x)-\lambda_{1} v(x), \quad x \in \Omega \text { a.e. }
$$

with $v \in D(A)=H^{2}(\Omega) \cap H_{0}^{1}(\Omega)$. Then we know that $A$ is self-adjoint and nonnegative, (H3)(1) is satisfied, and $H_{1}:=D\left(A^{1 / 2}\right)=H_{0}^{1}(\Omega)$. Set

$$
f(v)(x)=|v(x)|^{p} v(x), \quad v \in H_{1} .
$$

Using (5.3) gives

$$
\|f(v)\|=\|v\|_{L^{2 p+2}}^{1+p} \leq c_{2}\|v\|_{H^{1}}^{1+p}, \quad \forall v \in H_{1},
$$

with a constant $c_{2}>0$. So (H3)(2) holds. Moreover, (H1)(ii) and (H2)(1)-(2) hold too (cf. [18, the proof of Thm. 4.1] for details). Accordingly, Theorems 2.2 and 2.3 are applicable to problem (5.2), and so for the mild solution $u(t)$ and the energy

$$
E_{u}(t):=\frac{1}{2} \int_{\Omega}\left(\left|u_{t}\right|^{2}+|\nabla u|^{2}-\lambda_{1}|u|^{2}\right) \mathrm{d} x+\frac{1}{p+2}\left(\int_{\Omega}|u(t, x)|^{p+2} \mathrm{~d} x\right)
$$

of the problem (5.2), we have the same conclusions as in Example 5.1.

Acknowledgements. The authors would like to thank the referees very much for their truly professional and valuable comments and suggestions.

\section{REFERENCES}

[1] P. Acquistapace and B. Terreni, Infinite-horizon linear-quadratic regulator problems for nonautonomous parabolic systems with boundary control. SIAM J. Control Optim. 34 (1996) 1-30.

[2] N. Anantharaman and M. Léautaud, Sharp polynomial decay rates for the damped wave equation on the torus. Anal. PDE 7 (2014) 159-214. (With an appendix by Stéphane Nonnenmacher)

[3] E.R. Aragão-Costa, T. Caraballo, A.N. Carvalho and J.A. Langa, Non-autonomous Morse decomposition and Lyapunov functions for dynamically gradient processes. Trans. Am. Math. Soc. 365 (2013) 5277-5312.

[4] M. Balti and R. May, Asymptotic for the perturbed heavy ball system with vanishing damping term. Evol. Equ. Control Theory 6 (2017) 177-186.

[5] C.J.K. Batty, A. Borichev and Y. Tomilov, $L^{p}$-tauberian theorems and $L^{p}$-rates for energy decay. J. Functional Anal. 270 (2016) 1153-1201.

[6] N. Burq and R. Joly, Exponential decay for the damped wave equation in unbounded domains. Commun. Contemp. Math. 18 (2016) 1650012.

[7] A. Cabot and P. Frankel, Asymptotics for some semilinear hyperbolic equations with non-autonomous damping. J. Differ. Equ. 252 (2012) 294-322.

[8] A.N. Carvalho and J.A. Langa, The existence and continuity of stable and unstable manifolds for semilinear problems under non-autonomous perturbation in Banach spaces. J. Differ. Equ. 233 (2007) 622-653.

[9] A.N. Carvalho, J.A. Langa and J.C. Robinson, Attractors for infinite-dimensional non-autonomous dynamical systems. In vol. 182 of Applied Mathematical Sciences. Springer, New York (2013).

[10] M. Daoulatli, Rates of decay for the wave systems with time-dependent damping. Discrete Contin. Dyn. Syst. 31 (2011) 407-443.

[11] G. Dore, A. Favini, R. Labbas and K. Lemrabet, An abstract transmission problem in a thin layer, I: Sharp estimates. J. Functional Anal. 261 (2011) 1865-1922. 
[12] H.O. Fattorini, The Cauchy Problem. With a Foreword by Felix E. Browder, reprint of the 1983 original, In Vol. 18 of Encyclopedia Mathematics and its Applications. Cambridge University Press, Cambridge (2008).

[13] A. Favini, Degenerate and singular evolution equations in Banach space. Math. Ann. 273 (1985) 17-44.

[14] A. Favini, C.G. Gal, G.R. Goldstein, J.A. Goldstein and S. Romanelli, The non-autonomous wave equation with general Wentzell boundary conditions. Proc. Roy. Soc. Edinburgh Sect. A 135 (2005) 317-329.

[15] A. Favini and G. Marinoschi, Degenerate Nonlinear Diffusion Equations. Vol. 2049 of Lecture Notes in Mathematics. Springer, Heidelberg (2012).

[16] J.A. Goldstein, Time dependent hyperbolic equations. J. Functional Anal. 4 (1969) 31-49.

[17] J.A. Goldstein, Semigroups of Linear Operators and Applications. Oxford Mathematical Monographs, Oxford University Press, New York (1985).

[18] M. Ghisi, M. Gobbino and A. Haraux, Optimal decay estimates for the general solution to a class of semil-linear dissipative hyperbolic eqiations. J. Eur. Math. Soc. (JEMS) 18 (2016) 1961-1982.

[19] M. Ghisi, M. Gobbino and A. Haraux, Finding the exact decay rate of all solutions to some second order evolution equations with dissipation. J. Functional Anal. 271 (2016) 2359-2395.

[20] A. Haraux, Slow and fast decay of solutions to some second order evolution equations. J. Anal. Math. 95 (2005) $297-321$.

[21] A. Haraux, Decay rate of the range component of solutions to some semilinear evolution equations. NoDEA Nonlinear Differ. Equ. Appl. 13 (2006) 435-445.

[22] K.P. Jin, J. Liang and T.J. Xiao, Coupled second order evolution equations with fading memory: Optimal energy decay rate. J. Differ. Equ. 257 (2014) 1501-1528.

[23] K.P. Jin, J. Liang and T.J. Xiao, Uniform stability of semilinear wave equations with arbitrary local memory effects versus frictional dampings. J. Differ. Equ. 266 (2019) 7230-7263.

[24] P.E. Kloeden and M. Rasmussen, Vol. 176 of Nonautonomous Dynamical Systems. Mathematical Surveys and Monographs. American Mathematical Society, Providence, RI (2011).

[25] J.A. Langa, J.C. Robinson, A. Suárez and A. Vidal-López, The stability of attractors for non-autonomous perturbations of gradient-like systems. J. Differ. Equ. 234 (2007) 607-625.

[26] J. Liang, R. Nagel and T.J. Xiao, Approximation theorems for the propagators of higher order abstract Cauchy problems. Trans. Am. Math. Soc. 360 (2008) 1723-1739.

[27] J.L. Lions, Controlabilité exacte, perturbations et stabilisation de systèmes distribués 1, Masson, Paris (1988).

[28] J.R. Luo and T.J. Xiao, Decay rates for second order evolution equations in Hilbert spaces with nonlinear time-dependent damping. Evol. Equ. Control Theory 9 (2020) 359-373.

[29] P. Martinez, A new method to obtain decay rate estimates for dissipative systems. ESAIM: COCV 4 (1999) $419-444$.

[30] P. Martinez, Precise decay rate estimates for time-dependent dissipative systems. Israel J. Math. 119 (2000) $291-324$.

[31] R. May, Long time behavior for a semilinear hyperbolic equation with asymptotically vanishing damping term and convex potential. J. Math. Anal. Appl. 430 (2015) 410-416.

[32] M. Nakao, On the time decay of solutions of the wave equation with a local time-dependent nonlinear dissipation. Adv. Math. Sci. Appl. 7 (1997) 317-331.

[33] T.J. Xiao and J. Liang, Coupled second order semilinear evolution equations indirectly damped via memory effects. J. Differ. Equ. 254 (2013) 2128-2157.

[34] T.J. Xiao and J. Liang, Nonautonomous semilinear second order evolution equations with generalized Wentzell boundary conditions. J. Differ. Equ. 252 (2012) 3953-3971.

[35] T.J. Xiao and J. Liang, Vol. 1701 of The Cauchy Problem for Higher Order Abstract Differential Equations. Lecture Notes in Mathematics. Springer, Berlin (1998). 